\title{
The coincidence effect in similarity and choice
}

\author{
AUDREY S. KAPLAN and DOUGLAS L. MEDIN \\ Northwestern University, Evanston, Illinois
}

\begin{abstract}
Medin, Goldstone, and Markman (1995) recently described a series of parallel effects in similarity and choice. They suggested that similarity and choice are related in a nontrivial way such that choice may entail a similarity judgment to an explicit or constructed ideal. In this paper, the correspondences between similarity and choice were investigated with respect to a phenomenon in similarity known as the coincidence effect. In coincidence (pronounced "coincide-ence"), two items that match on one dimension but have a large difference on another dimension receive a higher similarity rating than do two items that have only modest differences on both dimensions. We conducted five experiments in order to examine commonalities between similarity and choice processes with respect to coincidence. Four types of tasks were given: similarity ratings, desirability ratings, forced choice similarities (which of two items is most similar to a target), and forced choice preferences (which of two items one would prefer, given a target). We found a main effect for ratings as opposed to forced choices, with ratings showing greater coincidence effects than did choices. Similarity measures tended to produce more coincidence than did preference measures. The overall pattern of results suggests the presence of dimensional weighting processes sensitive to task characteristics and operating somewhat differently for similarity and decision making.
\end{abstract}

Similarity judgments and decision making are two of the most often studied areas in cognitive psychology, yet they are rarely considered together. In a recent analysis of the literature from both domains, Medin, Goldstone, and Markman (1995) highlighted a number of parallels between them. Medin et al. proposed that such surface level correspondences between similarity processing and decision making might be based on deeper commonalities between these two domains. In addition, they speculated that decision making may actually entail a similarity judgment to an explicit or constructed ideal. One way to explore this possibility is to find a phenomenon in one domain and look for a parallel to it in the other. Even if the effect fails to transfer, the pattern of differences that is revealed may provide helpful clues toward determining the nature of the relationship between similarity and choice.

The present research examines a phenomenon in similarity known as the coincidence effect, which seems closely linked to comparison with standards. Tversky and Gati (1982) first reported the coincidence effect whereby identity on a dimension is overweighed in similarity and dissimilarity judgments. For example, in Figure 1, items represented by $\mathrm{A}$ and $\mathrm{C}$ are rated as more similar to $\mathrm{T}$ than $B$ is, even though, in metric terms, $B$ is closer to $T$. The logic of coincidence is that exact matches on a dimension

Preparation for this article was supported by National Science Foundation Grant 92-11277. We thank Evan Heit, Alexander Aminoff, and an anonymous reviewer for valuable advice and suggestions. The research described in this article conforms with the American Psychological Association ethical standards for research involving human participants. Correspondence concerning this article should be addressed to A. S. Kaplan, who is now at the University of Illinois, at $603 \mathrm{E}$. Daniel St., Champaign, IL 61820 (e-mail: akaplan@s.psych.uiuc.edu). are weighted more heavily than overall proximity in a similarity space. Tversky and Gati obtained reliable coincidence effects for both perceptual and conceptual stimuli.

There are situations in decision making wherein identity to ideals seems to be preferentially weighted. An example is the certainty effect (Tversky \& Kahneman, 1981). Given the choice between an $80 \%$ chance to win $\$ 40$ and a $100 \%$ chance to win $\$ 30$, most people choose the latter option even though the expected value is higher for the former ( $\$ 32$ vs. \$30). One explanation of this phenomenon is that the certainty of winning the $\$ 30$ outweighs the extra $\$ 2$ because people worry about how much they would regret having taken the first bet and losing when they could have had $\$ 30$ for sure. In this case, the certain probability can be seen as matching the ideal of "maximum chance of winning" (the other dimension is dollar value, which presumably also has a maximum ideal). However, this may not be a legitimate example of coincidence, because probability and amount are atypical dimensions with rigid ideal values. More to the point, there are alternative explanations of the certainty effect, such as loss aversion. Simply put, loss aversion means that potential losses are weighed more heavily than potential gains in relation to some reference point. According to this account, the possible loss (i.e., the $20 \%$ chance of winning nothing) is avoided when one has a sure thing.

Still other results in decision making seem directly opposed to coincidence. Simonson and Tversky (1992) describe a phenomenon in choice, extremeness aversion, whereby people prefer an item with two small disadvantages to one with a single, but large, disadvantage. Consider Figure 1 again: extremeness aversion predicts that people will choose $\mathrm{B}$ over $\mathrm{A}$ and $\mathrm{C}$ when $\mathrm{T}$ is the target, because even though $\mathrm{A}$ and $\mathrm{C}$ coincide with $\mathrm{T}$ on one di- 


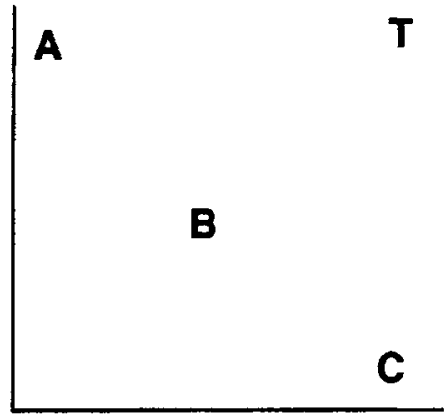

Figure 1. The coincidence effect says that $A$ and $C$ will be rated as more similar to $T$ than $B$ because they match $T$ on one dimension each while $B$ does not match $T$ on either dimension. In metric terms, $B$ is closer to $T$.

mension, they differ greatly on the other dimension. Option $\mathrm{B}$ can be seen as a compromise between $\mathrm{A}$ and $\mathrm{C}$. However, when one is rating similarity, the coincidence effect shows that $\mathrm{A}$ and $\mathrm{C}$ are considered more similar to $\mathrm{T}$ than is $\mathrm{B}$. This pair of results seems troubling for the hypothesis that choice and similarity involve similar processes. How can similarity and choice share common processes if they give rise to incompatible effects? One possibility is that there are different kinds of, or respects for, similarity (Medin, Goldstone, \& Gentner, 1993). Thus, some conditions may lend themselves to coincidence, whereas others favor anticoincidence (as in extremeness aversion). In particular, similarity and choice were measured differently in the examples above; similarity was rated on a numerical scale and the choices were binary preferences. It is unclear what would happen if we asked people to rate their preferences numerically or make similarity judgments as forced choices.

Choice is known to embody flexibility, as has been well witnessed by context effects. For example, preference reversals occur when different ways of assessing preferences yield different results. Slovic and Lichtenstein (1968) reported that people would rather play a gamble with a high probability of winning, but that they would pay more to play, or ask a higher selling price for, a gamble with a larger payoff. Here the dimensions of payoff and probability are weighted differently, depending on the context.

The experiments in this paper were performed in an attempt to unconfound similarity from ratings and preference from forced choice. By so doing, we hoped to discover whether there are conditions under which similarity and choice yield the same results, or whether they are systematically different. In the first two experiments, we were concerned with ratings; in the first, participants made similarity ratings, and in the second, participants made desirability ratings. In the third and fourth experiments, we employed a forced choice task in which participants had to choose which of two options was more similar to a target (Experiment 3) or which option they would prefer, relative to the same target (Experiment 4). Finally, in the fifth experiment we examined the effects of explicit dimensional weighting strategies (or lack thereof) on the coincidence effect.

To our knowledge, no one has either replicated the coincidence effect or related it to forced choice. The goal of the present study was to determine the range of contexts in which the coincidence effect would appear. Specifically, we were interested in two factors of the task. First, is the coincidence effect dependent on ratings of similarity, or would desirability (preference) ratings show a coincidence pattern as well? Second, do people ever employ a coincidence strategy in forced choices? The forced choice results of Simonson and Tversky (1992) suggest that they do not. Their choices were preferences, however, and it is not clear how coincidence would fare if people were asked to choose which of two options were most similar to a target. In Experiments 3 and 4, similarity forced choices were contrasted with preference forced choices. Thus, there were two kinds of judgments to be made, similarity and preference, and two modes of responding, ratings or forced choices. The experiments that follow addressed each of these situations in turn.

\section{EXPERIMENTS 1-2 Similarity and Desirability Ratings}

Tversky and Gati (1982) originally discovered the coincidence effect by using perceptual stimuli, but they also obtained the effect with conceptual materials. Their conceptual stimuli were descriptions of students on two dimensions: academic major (ordered from technical to humanistic) and political affiliation (ordered from conservative to liberal). The conceptual stimuli used in our experiments were brief descriptions of dogs, containing information only on their size and temperament levels. The sizes used were very large, large, medium, and small; the temperament levels were sociable to any person, friendly, tolerant, and somewhat tolerant. The purpose of Experiment 1 was to replicate the findings of Tversky and Gati. Here, we asked participants to rate the similarity of all possible pairs of different dogs. In Experiment 2, participants were asked to rate the desiribility of an available dog given an ideal dog. The ideal and available dog pairs were exactly those used in Experiment 1. We expected to find a coincidence effect in Experiment 1, but it was unclear whether desirability ratings would also exhibit a coincidence effect. Given the loss aversion results discussed above, it may be that preferences do not exhibit coincidence.

\section{Method}

Subjects. Twenty-six college age people residing in the Chicago metropolitan area participated in each study. In all the experiments reported in this paper, nobody participated in more than one study. Participation was solicited by advertisements on the Northwestern University campus. Participants were paid for their cooperation.

Materials. The stimuli for all experiments consisted of brief descriptions of dogs characterized on two dimensions: size and temperament. Four levels of each dimension were specified. The sizes were, in increasing order: small, medium, large, and very large. The temperament levels were, in increasing ferocity: sociable to any 
person, friendly, tolerant, and somewhat tolerant. Figure 2 shows the 16 different dog descriptions generated by pairing each size with each temperament.

Procedure. All experiments were run on a computer with a 23.5 $\times 17.5 \mathrm{~cm}$ display screen. In Experiment 1 , participants were told that they would see a series of dog descriptions, based on size and temperament, two at a time. Their task was to rate the similarity of the two dogs on a scale of $1-9$, with 1 corresponding to the lowest similarity rating and 9 to the highest. Participants were told about all of the possible sizes and temperaments in the instructions in order to ensure that they took into consideration the combinations they were likely to see later when making their early judgments. All 120 possible dog description pairs were presented. The experiment lasted approximately $20 \mathrm{~min}$.

The dog descriptions were written in boxes $(6.8 \times 2.8 \mathrm{~cm})$ displayed on the screen $0.5 \mathrm{~cm}$ apart. The size was written on the first line, and the temperament was written directly below it. Buttons ranging from 1 to 9 were placed under the descriptions with the words "low sim." under the low end of the scale and "high sim." under the high end of the scale. Participants clicked on the rating buttons to indicate the similarities. The order of dog description presentations was randomized. Participants were self-paced and were debriefed at the end of the experiment.

In Experiment 2, participants read the following instructions.

In this experiment we would like you to pretend that you are the owner of a pet store that sells various types of dogs. Customers will come in and describe to you the size and temperament characteristics of their ideal dog choices. You will see a series of dog descriptions, two at a time. One will be the customer's ideal and the other will be a dog that you have available for sale. Each dog will be described by a size: small, medium, large, or very large, and a temperament: sociable to any person, friendly, tolerant, or somewhat tolerant. For each pair, we would like you to rate the desirability of the available dog given the ideal dog on a nine point scale where one is low desirability and nine is high desirability.

To ensure that each dog appeared an equal number of times as the ideal and available dog, participants did each desirability rating twice, once with the ideal on the left and the available on the right, and once vice versa. Thus, each participant produced 240 desirability ratings. The experiment lasted approximately $40 \mathrm{~min}$. Participants were debriefed at the end of the experiment.

\section{Results and Discussion}

The data were analyzed in two different ways. Following Tversky and Gati (1982), we analyzed all quadruples

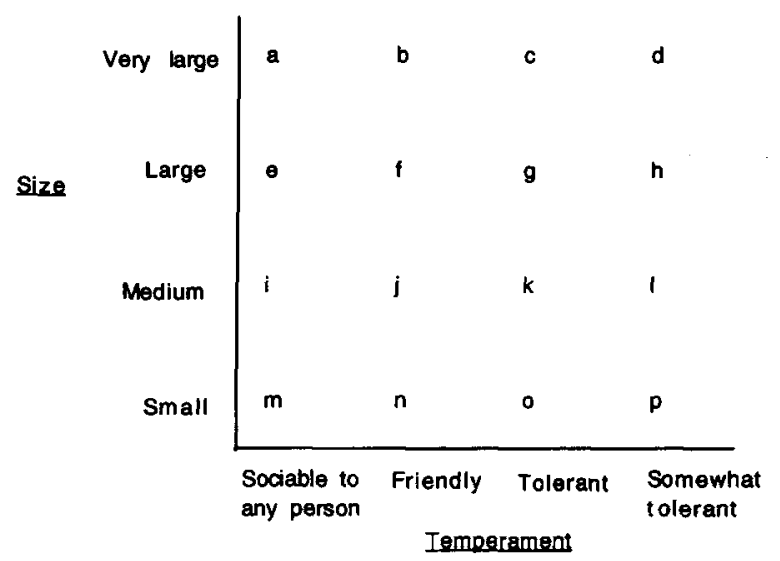

Figure 2. Stimuli for the experiments. Each lowercase letter represents a dog description (i.e., $a=$ very large, sociable to any person). forming the three corners of a right triangle plus a point on the hypotenuse (see Figure 3). The triangle inequality states that the sum of any two sides of a triangle must be greater than the third side. Since similarity is inversely proportional to distance (i.e., two things rated highly similar are very close in a similarity space), one can see that the triangle inequality is satisfied when

$$
\operatorname{sim}(j, b) \leq \operatorname{sim}(b, g) \text { and } \operatorname{sim}(j, l) \leq \operatorname{sim}(g, l)
$$

or

$$
\operatorname{sim}(j, b) \leq \operatorname{sim}(g, l) \text { and } \operatorname{sim}(j, l) \leq \operatorname{sim}(b, g) .
$$

The triangle inequality is violated when the opposite pattern of inequalities holds. Another way of stating the triangle inequality is to say that the corner path from $b$ to $l$ (going through $j$ ) must be greater than the center path from $b$ to $l$ (going through $g$ ). Coincidence can be thought of as a violation of the triangle inequality, because if dimensional matches are overweighed in similarity judgments, the legs of the triangle become shorter, diminishing the corner path.

There were 24 appropriate triangles in our stimulus set: 4 from each of the four $3 \times 3$ subsquares plus 2 from each of the four extreme corners. The hypotenuse stimulus on each of the extreme triangles was always one of the two points along the diagonal of that triangle. For example, when $a$ was the corner, the diagonals were $d, g, m$ and $d, j, m$, where $g$ and $j$ were the hypotenuse stimuli. Thus, each extreme corner right angle was used in two triangles, one with each of the hypotenuse stimuli as the center.

For each participant, we counted $\mathrm{Co}$, the number of triangles for which the corner path was greater than the center path (confirming the triangle inequality), and $\mathrm{Ce}$, the number of triangles for which the center path was greater than the corner path (indicating coincidence). Participants were then grouped into the following five categories: (1) $\mathrm{Co} \gg \mathrm{Ce}$ (double inequality sign indicates significance by sign test at the .05 level); (2) $\mathrm{Co}>\mathrm{Ce}$; (3) $\mathrm{Co}=\mathrm{Ce}$; (4) $\mathrm{Co}<\mathrm{Ce}$; and (5) $\mathrm{Co} \ll \mathrm{Ce}$.

We also analyzed the data using an ideal-based method. Each right-angle corner stimulus can be thought of as an ideal. For example, in Figure 3, where the ideal is $j$, we compared $\operatorname{sim}(j, b)$ with $\operatorname{sim}(j, g)$ and $\operatorname{sim}(j, l)$ with $\operatorname{sim}(j, g)$. If the similarity of the ideal to a coinciding dog is greater than the similarity of the ideal to the hypotenuse dog, we have a case of coincidence. The opposite relation is an example of anticoincidence. For each participant we counted $\mathrm{A}$, the number of incidents of anticoincidence, and $\mathrm{C}$, the number of coincidence occurrences. In this analysis, there were 48 comparisons instead of 24 , because each ideal is compared with the coinciding alternative on each dimension separately. Participants were then grouped into the following five categories: (1) $\mathrm{A} \gg \mathrm{C}$ (double inequality sign indicates significance by sign test at the .05 level); (2) $\mathrm{A}>\mathrm{C}$; (3) $\mathrm{A}=\mathrm{C}$; (4) $\mathrm{A}<\mathrm{C}$; and (5) $\mathrm{A} \ll \mathrm{C}$. Note that $A$ is analogous to $C o$ and $C$ is analogous to $C e$ in that they are measures of a participant's tendency toward anticoincidence and coincidence, respectively. Thus, 


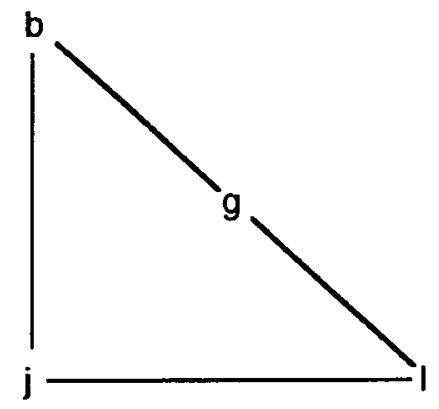

Figure 3. An example of a triangle used to test the triangle inequality and ideal-based coincidence.

we should find roughly equivalent numbers of participants in corresponding categories across these two classification systems.

We used the triangle inequality method because this is how Tversky and Gati (1982) analyzed their results and we wanted to be able to compare our results with theirs. The ideal-based method was used because it allowed us to directly test the hypothesis that forced choices are based on similarity to an ideal. For example, in Figure 3, if $\operatorname{sim}(j, b)$ were greater than $\operatorname{sim}(j, g)$, we might expect that $b$ would be chosen over $g$ when $j$ was the ideal.

Table 1 contains the results from all five experiments. The results of both methods of analysis show that coincidence was the dominant strategy employed by our participants in Experiment 1. In both cases, 23 out of 26 participants showed a coincidence pattern more often than anticoincidence, and no participants were in the statistically significant anticoincidence groups. If for each method of analysis we collapse the two groups above the equality relation $(\mathrm{Co} \gg \mathrm{Ce}$ and $\mathrm{Co}>\mathrm{Ce}$ for the triangle inequality method and $\mathrm{A} \gg \mathrm{C}$ and $\mathrm{A}>\mathrm{C}$ for the idealbased method), we derive two groups corresponding to overall anticoincidence. Doing the same for groups below the equality relation gives us two overall coincidence groups. This redistributes our participants into two categories, overall coincidence (OC) or overall anticoincidence $(\mathrm{OA})$.

Having done this, we compared the number of participants in the $\mathrm{OC}$ and OA groups. Within each method of analysis, a statistically reliable coincidence effect was found in Experiment 1 . For the triangle inequality method, $\chi^{2}(1, N=26)=15.38, p<.01$. For the idealbased method, $\chi^{2}(1, N=25)=17.64, p<.01$. We went back to Tversky and Gati's (1982) triangle inequality study on students and reorganized their data in this fashion and found $\chi^{2}(1, N=29)=3.90, p<.05$ in favor of coincidence. In short, we replicated Tversky and Gati's coincidence effect.

The results from Experiment 2 were more ambiguous. The participants in this experiment failed to show predominantly coincidence or anticoincidence, as evidenced by the $\mathrm{OA}$ and $\mathrm{OC}$ groupings for the triangle inequality method (13 and 13) and the ideal-based method (10 and 15 , respectively). About half of the participants favored a coincidence pattern, and about half favored an antico- incidence pattern. A chi-square test on the $\mathrm{OA}$ and $\mathrm{OC}$ groups between Experiments 1 and 2 showed that the coincidence effect is reliably larger in Experiment $1\left[\chi^{2}(1\right.$, $N=50)=7.02, p<.01]$.

Finally, for each participant we computed the proportion coincidence (PC) according to the formula $\mathrm{PC}=\mathrm{Ce} /$ $(\mathrm{Co}+\mathrm{Ce})$ for the triangle inequality method and $\mathrm{PC}=$ $\mathrm{C} /(\mathrm{A}+\mathrm{C})$ for the ideal-based method. The mean PCs for Experiment 1 (mean $\mathrm{PC}=.76, S D=.18$ for the triangle inequality method, and mean $\mathrm{PC}=.69, S D=.16$ for the ideal-based method) were higher than those for Experiment 2 (mean $\mathrm{PC}=.51, S D=.28$ for the triangle inequality method, and mean $\mathrm{PC}=.51, S D=.14$ for the ideal-based method). The ideal-based PC values are significantly different from .5 only in Experiment $1[t(25)=$ $7.22, p<.001$ for the triangle inequality, and $t(25)=5.94$, $p<.001$ for the ideal-based], again indicating coincidence.

Overall then, the similarity ratings reveal a robust coincidence effect, whereas the desirability ratings of exactly the same pairs do not. On the other hand, the desirability ratings also do not show the anticoincidence pattern noted by Simonson and Tversky (1992). One interpretation of the difference between similarity and desirability rating results is that the desirability task strongly encourages an equal weighting of dimensions. If so, one should be able to reduce or eliminate the coincidence effect in similarity judgments by instructions aimed at discouraging selective weighting of dimensions. Experiment 5 examines this possibility. First, however, we turn our attention to forced choice measures of similarity and preference.

\section{EXPERIMENTS 3-4 Forced Choice Similarity and Preference}

Imagine a forced choice situation in which one is presented with a target dog-say, "Very large, Tolerant"above two option dogs - say, "Very large, Sociable to any person" and "Large, Friendly." In the following two ex-

Table 1

Grouping of Participants in all Experiments

\begin{tabular}{lrrrrr}
\hline & \multicolumn{5}{c}{ Experiment } \\
\cline { 2 - 6 } Category & 1 & 2 & 3 & 4 & 5 \\
\hline & \multicolumn{5}{c}{ Triangle Inequality } \\
Co $\gg \mathrm{Ce}$ & 0 & 5 & - & - & 3 \\
$\mathrm{Co}>\mathrm{Ce}$ & 3 & 8 & - & - & 8 \\
$\mathrm{Co}=\mathrm{Ce}$ & 0 & 0 & - & - & 0 \\
$\mathrm{Co}<\mathrm{Ce}$ & 7 & 9 & - & - & 9 \\
$\mathrm{Co} \ll \mathrm{Ce}$ & 16 & 4 & - & - & 6 \\
& & Ideal-Based & & \\
$\mathrm{A} \gg \mathrm{C}$ & 0 & 3 & 5 & 19 & 1 \\
$\mathrm{~A}>\mathrm{C}$ & 2 & 7 & 12 & 6 & 8 \\
$\mathrm{~A}=\mathrm{C}$ & 1 & 1 & 2 & 0 & 1 \\
$\mathrm{~A}<\mathrm{C}$ & 9 & 12 & 5 & 1 & 10 \\
$\mathrm{~A} \ll \mathrm{C}$ & 14 & 3 & 6 & 0 & 6 \\
\hline $\mathrm{N}$ & & & & & \\
\hline
\end{tabular}

Note - Co, number of triangles for which the corner path was greater than the center path; $\mathrm{Ce}$, number of triangles for which the center path was greater than the corner path; A, number of incidents of anticoincidence; $\mathrm{C}$, number of incidents of coincidence. 
periments, we presented participants with such triads and asked them to choose which of two option dogs was most similar to the target dog (Experiment 3 ) or which of the same two option dogs they would prefer, given the target dog as an ideal (Experiment 4). Both of these tasks involve forced choices; the difference is that Experiment 3 was a similarity forced choice and Experiment 4 was a preference forced choice. Thus, Experiments 3 and 4 were forced choice analogues of Experiments 1 and 2, respectively.

If people use a coincidence strategy, we would expect them to choose the dog that is "Very large, Sociable to any person" over the dog that is "Large, Friendly," because the target and the first option match on the size dimension. The other dog would be picked more often in this triad if people used an anticoincidence strategy. We predicted that people would show anticoincidence in the preference task (Experiment 4), because it seems in line with the Simonson and Tversky (1992) results on extremeness aversion. However, it was unclear what to expect when we asked people to choose one of the dogs as being most similar to the target (Experiment 3 ). If the coincidence effect is not sensitive to response mode (i.e., ratings vs. forced choices), we would expect results similar to those of Experiment 1. If a strong coincidence pattern is dependent on numerical ratings, we would expect the opposite pattern of results.

\section{Method}

Subjects. Twenty-six Northwestern University undergraduates participated in each experiment. Most participated to fulfill a class requirement, but some were paid for their participation.

Materials. The same dog descriptions that were used in the last experiment were used as stimuli in this experiment.

Procedure. The instructions for Experiment 3 read as follows:

You will see a series of dog descriptions three at a time. Each dog will be described by a size and a temperament. The sizes are, in increasing order: small, medium, large, and very large. The temperament levels are, in increasing ferocity: sociable to any person, friendly, tolerant, and somewhat tolerant. In each case the dog at the top of the screen will be the "Target Dog." Your task is to choose which of the two dogs underneath is more similar to the "Target Dog." Just click on the button underneath the dog of your choice.

The instructions for Experiment 4 read as follows:

In this experiment we would like you to pretend that you are the owner of a pet store that sells various types of dogs. Customers will come in and describe to you the size and temperament characteristics of their ideal dog choices. Along with the customer's ideal, you will see two $\operatorname{dog}$ choices (labeled $A$ and $B$ ) that you have for sale. Each $\operatorname{dog}$ will be described by a size and a temperament. The sizes are, in increasing order: small, medium, large, and very large. The temperament levels are, in increasing ferocity: sociable to any person, friendly, tolerant, and somewhat tolerant. For each pair of available dogs we would like you to recommend one of the dogs to the customer based on their stated ideal. Just click on the corresponding button (A or B) underneath your dog recommendation.

For each triad, the target (same as customer's ideal) was a corner stimulus of a $3 \times 3$ subset of the stimuli in Figure 2 or a corner of the entire set ( $\operatorname{dogs} a, d, m$, and $p$ ). Thus, the targets corresponded to the ideals from the ideal-based method of analysis. The option dogs were two of the three dogs forming the diagonal of a given subset consisting of the hypotenuse (anticoincidence) stimulus plus one of the coinciding dogs. For example, when $\operatorname{dog} f$ was the target, the options were $h$ and $k$ on one trial and $k$ and $n$ on another. Thus for each $3 \times 3$ subsquare, two pairs of options were presented (on separate trials). Notice that $k$ differs from $f$ on two dimensions, whereas $h$ and $n$ coincide with $f$ on size and temperament, respectively. In this way, the participant was always choosing between the coinciding and anticoinciding dogs (Experiment 4) or choosing the more similar of a coinciding and anticoinciding dog to the target (Experiment 3). For each $4 \times 4$ target, two diagonals were used, consisting of the two coinciding extremes with one of the two intermediates along the diagonal (e.g., target $a$ with $m, j$, and $d$, and with $m, g$, and d). Thus for each $4 \times 4$ target, four pairs of options were presented (on separate trials). The triads were shown in random order. Both experiments lasted about $15 \mathrm{~min}$. Participants were self-paced and were debriefed at the end of the experiment.

\section{Results and Discussion}

Since in each trial of these two experiments the participant chose either the coinciding or the anticoinciding $\mathrm{dog}$, we counted A and C directly. The results from Experiment 3 showed a modest but reliable tendency toward an anticoincidence pattern. Out of 26 participants, 17 showed an anticoincidence pattern more often than not, and 7 showed a coincidence pattern a majority of the time. This result is statistically significant $\left[\chi^{2}(1, N=24)=4.16\right.$, $p<.05]$. The mean PC value $(.45, S D=.15)$, while not reliable, is also in the direction of anticoincidence.

In Experiment 4, 25 out of 26 participants showed an anticoincidence pattern rather than coincidence, 19 of them significantly more often. This anticoincidence effect was highly significant when we compared the overall anticoincidence and overall coincidence collapsed groups $\left[\chi^{2}(1, N=26)=11.08, p<.01\right]$. The mean PC value for Experiment 4 was $.25(S D=.15)$. This value is significantly different from $.5[t(25)=8.33, p<.001]$, indicating anticoincidence. This result is compatible with extremeness aversion.

The combined results of Experiments 3 and 4 suggest that anticoincidence is the dominant pattern in forced choice situations. However, if we compare the OA and OC groups across these two experiments, we find that the anticoincidence effect is larger in forced choice preferences than in forced choice similarity $\left[\chi^{2}(1, N=\right.$ $50)=5.95, p<.05]$. Looking across Experiments 1 and 2 in comparison with 3 and 4 , one can see both a main effect of task (ratings lead to more coincidence; choices, to anticoincidence) and a main effect of similarity versus preference (with similarity leading to larger coincidence effects). We believe that both patterns can be accommodated within the framework of factors that promote or discourage differential weighting of dimensions. In the final experiment, we examined the role of selective attention in similarity ratings. We hypothesized that we would see an anticoincidence or a very weak coincidence effect if people were encouraged to pay attention to both dimensions.

\section{EXPERIMENT 5}

In Experiment 5, participants were asked to pretend that they were the owner of a pet store selling various types of dogs described by the same size and tempera- 
ment levels as in the previous experiments. They were told that they would see pairs of dog descriptions, one of which was labeled as the customer's ideal and the other labeled as a dog available for sale. The task was to rate the similarity of the available dog to the ideal dog on a scale of 1 to 9 . Thus, this task can be seen as a hybrid of Experiments 1 and 2. However, unlike in the earlier tasks, the instructions in this experiment stressed the importance of paying attention to both dimensions.

\section{Method}

Subjects. Twenty-six college-age people residing in the Chicago metropolitan area participated in this study. Participation was solicited by advertisements on the Northwestern University campus Participants were paid for their cooperation.

Materials. The same dog descriptions used in the previous studies were used in this experiment as well.

Procedure. In this experiment, participants were asked to pretend that they were the owner of a pet store selling various types of dogs described by the same size and temperament levels as in the first two experiments. They were told that they would see pairs of dog descriptions, one of which was labeled as the customer's ideal and the other labeled as a dog available for sale. The labels were written underneath the boxes containing the dog descriptions. The task was to rate the similarity of the available dog to the ideal dog on a scale of $1-9$. The instructions stressed the importance of paying attention to both dimensions with the following statement: "Both dimensions (size and temperament) are equally important. For example, someone wanting a guard dog for a small apartment would need a dog which was both small and somewhat tolerant as opposed to one which was very large and friendly." To ensure that each dog appeared an equal number of times as the ideal and available dog, participants did each similarity rating twice, once with the ideal on the left and the available on the right and once vice versa. Thus each participant produced 240 similarity ratings. Participants were self-paced, and the experiment lasted approximately $40 \mathrm{~min}$. Participants were debriefed at the end of the experiment.

\section{Results and Discussion}

The data were analyzed using the triangle inequality and ideal-based methods as in the previous experiments. Consistent with our hypothesis, the similarity rating results were quite different from those of Experiment 1. In the triangle inequality groupings, only 15 out of 26 participants showed a coincidence pattern rather than anticoincidence. In the ideal-based groupings, 16 out of 26 participants showed a coincidence pattern. The distribution of participants across these groups was nearly equal, as is indicated by the number of people in the OA and OC groups, the difference of which failed to reach statistical significance $\left[\chi^{2}(1, N=26)=0.62, p>.05\right.$ for the triangle inequality method, and $\chi^{2}(1, N=25)=1.96$, $p>.05$ by the ideal-based method].
Although one must be cautious in making cross-experiment comparisons, it appears that the instructions to attend to both dimensions diminished the coincidence effect. Comparing the number of participants in the $\mathrm{OA}$ and $\mathrm{OC}$ groups between experiments shows that more people showed a coincidence pattern in Experiment 1 than in Experiment $5\left[\chi^{2}(1, N=52)=6.25, p<.02\right.$ for the triangle inequality method, and $\chi^{2}(1, N=50)=$ $5.73, p<.02$ for the ideal-based method].

In addition, the mean PCs for Experiment 1 (mean PC = $.76, S D=.18$ for the triangle inequality method, and mean $\mathrm{PC}=.69, S D=.16$ for the ideal-based method) were higher than those for Experiment 5 (mean PC $=.58$, $S D=.36$ for the triangle inequality method, and mean $\mathrm{PC}=.59, S D=.18$ for the ideal-based method). The ideal-based $P C$ values are significantly different from .5 in both experiments [for Experiment 1, $t(25)=6.15, p<$ .001 ; for Experiment $5, t(25)=2.464, p<.02]$, indicating coincidence. The mean PC values for Experiments 2 and 5 , however, are not reliably different $[t(50)=.77$, n.s. $]$.

Overall, the combination of specifying an ideal and explicitly asking participants to pay equal attention to dimensions considerably reduced or eliminated the coincidence effect. The similarity of these results to those of Experiment 2 suggests that desirability ratings may inherently employ a process of equal weighting of dimensions. These results support our hypothesis that selective attention plays an important role in the coincidence effect. As we have seen in Experiments 3 and 4, this selective attention strategy does not operate in forced choice situations.

\section{GENERAL DISCUSSION}

Table 2 summarizes the results from each experiment. It is evident from our results that participants show different similarity strategies (i.e., coincidence or anticoincidence) to different degrees, depending on the task. The picture that emerges suggests that coincidence is just one type of similarity pattern that people produce when comparing stimuli composed of two separable dimensions. More specifically, coincidence is the dominant pattern in numerical ratings tasks and anticoincidence is favored in choice contexts. This is captured nicely in the coincidence result from Experiment 1 and the trends toward coincidence in Experiments 2 and 5, all of which required numerical ratings of our stimuli, and the anticoincidence results Experiments 3 and 4, which were choice tasks involving the exact same items.

Table 2

Summary of Results

\begin{tabular}{|c|c|c|c|}
\hline Experiment & Presentation & Task & Result \\
\hline 1 & similarity & ratings & strong coincidence \\
\hline 2 & desirability & ratings & $50 / 50$ \\
\hline 3 & similarity & foreed choice & weak anticoincidence \\
\hline 4 & preference & forced choice & strong anticoincidence \\
\hline 5 & similarity & ratings/no selective attention & weak coincidence \\
\hline
\end{tabular}


Coincidence and anticoincidence can be thought of as poles on a continuum of differential weighting of dimensions. It is possible to construct situations in which people use strategies nearer to the middle of the continuum. In Experiment 5 , for example, the task was to rate the similarity of the two dogs on a scale of $1-9$, but the context was related to choice in that the cover story placed the participant in the position of a dog seller who helps people make choices. It is possible that these situational factors, along with the instructions to consider both dimensions, helped reduce the coincidence effect by moving people's strategies away from the extreme coincidence pole. The desirability rating task in Experiment 2 also appeared to diminish selective weighting of one dimension over the other, again reducing the coincidence effect. In short, coincidence is a malleable phenomenon. Our results suggest that selective attention may be responsible for the coincidence effect. Given the dramatic shift in the coincidence/anticoincidence results in ratings versus choice, it is not improbable that choice situations inherently employ a comparison process relying on more equal weighting of dimensions. ${ }^{1}$

If we think of coincidence and anticoincidence as qualitative indicators on a single dimension of differential weighting, our entire pattern of results can be interpreted within a relatively simple framework. Ratings tasks may promote selective weighting of matching dimensions. Both the implicit demands associated with desirability ratings and explicit instructions to attend to both dimensions appear to reduce selective weighting. The forced choice context appears to reduce selectivity to the point of yielding reliable anticoincidence effects.

It is important to note that we not only observe a main effect of ratings versus forced choice but also a main effect of similarity versus preference. Empirically, then, preference is not the same as similarity assessment, and our results fail to support the straightforward idea that preference is simply a similarity judgment (wherein the alternative selected is most similar to some ideal). Instead it appears that similarity judgments involve relatively more differential weighting of dimensions (or greater weight to identity on a dimension) than does preference. We suggest, however, that caution should be exercised with respect to generalizations at the level of similarity versus preference. For example, alternative ways of assessing similarity, such as asking which alternative is more different rather than more similar, may yield a different pattern of results (see Medin et al., 1995). Consequently, it is unclear whether some other similarity assessment procedure would yield levels of coincidence or anticoincidence that are quantitatively closer to those seen in preference. Proponents of parallels between similarity and decision making can take at least some comfort in the fact that ratings versus forced choice affect similarity and preference in the same way qualititatively.

Overall, our findings reinforce the strategy of exploring empirical parallels between similarity judgments and decision making and the utility of linking observations in the two domains. It is only by making such a comparison that one would note that coincidence effects and extremeness aversion are mirror-image (opposite) phenomena. Continuing in this vein, we can classify the difference between ratings and forced choice judgments as a preference reversal. Moreover, the choice-based measure of similarity agrees with the choice-based preference results in that they both produce anticoincidence patterns, although to different degrees. So it appears that, at least in some contexts, decision making could involve a similarity judgment to an explicit or constructed ideal. Advocates of this view would, however, need to explain why this common similarity process apparently involves less selective attention for decision making than for similarity judgment (compare Experiments 3 and 4). In conclusion, the present studies place the coincidence effect on firmer and more delimited empirical grounds and encourage further explorations of parallels (and nonparallels) between similarity judgments and decision making.

\section{REFERENCES}

Medin, D. L., Goldstone, R. L., \& Gentner, D. (1993). Respects for similarity. Psychological Review, 100, 254-278.

Medin, D. L., Goldstone, R. L., \& Markman, A. B. (1995). Comparison and choice: Relations between similarity processes and decision processes. Psychonomic Bulletin \& Review, 2, 1-19.

Simonson, I., \& TVersky, A. (1992). Choice in context: Tradeoff contrast and extremeness aversion. Journal of Marketing Research, 29, 281-295.

SLovic, P., \& Lichtenstein, S. (1968). The relative importance of probabilities and payoffs in risk taking. Journal of Experimental Psychology Monographs, 78 (3, Pt. 2).

TVERSKY, A., \& GATI, I. (1982). Similarity, separability, and the triangle inequality. Psychological Review, 89, 123-154.

TVERSKY, A., \& KaHNEMAN, D. (1981). The framing of decisions and the psychology of choice. Science, 185, 1124-1131.

\section{NOTE}

1. In addition to the experiments reported above, we conducted a separate study in which participants made two similarity ratings on each trial. We presented triads of dog descriptions identical to those from Experiments 3 and 4 , and the task was to rate the similarity of each of the nontarget dogs to the target. Recall that in these triads, one of the items to be compared with the target matched the target on one dimension but was quite different on the other dimension (we called this the coincider), and the other item (the anticoincider) mismatched on both dimensions but with differences that were only moderate. By comparing the similarity ratings given between the coincider and the anticoincider to the same target, we found that the coinciders were consistently rated more similar to the targets, in accord with the coincidence effect. Experiment 3 involved the same dog triads, but the participants had to choose which of the two nontargets was most similar to the target. In this case, people opted for the anticoincider a majority of the time. Therefore, we can assume that they were not comparing similarity assessments computed separately and independently for each of the two options. If they had been, we should have seen another coincidence effect in Experiment 3.

(Manuscript received February 16, 1996; accepted for publication April 28, 1996.) 